\title{
ERRATUM
}

Martin Zeller · Simone König

\section{The impact of chromatography and mass spectrometry on the analysis of protein phosphorylation sites}

Published online: 25 March 2004

(C) Springer-Verlag 2004

\section{Anal Bioanal Chem (2004) 378:898-909}

The following passage on page 901 contains minor printing errors. The correct text is:

The example of the phosphoprotein standard $\beta$-casein demonstrates some of the difficulties one might have to expect in phosphorylation site analysis. Only the smaller phosphopeptide (amino acid residues 33-48, FQpSEEQQQTEDELQDK, $m / z$ 2061.8290; SwissProt P02666) was detected, but was then missed by data-dependent HPLCMS/MS in positive ion mode. Only SIM on the pre-determined $\mathrm{m} / \mathrm{z}$ finally produced a suitable spectrum (Fig. 2). MALDI-MS is often used to identify phosphopeptide candidates for such specific fragmentation experiments [82]. The larger peptide (1-25, RELEELNVPGEIVEpSLpSpSpSEESITR, $m / z$ 3122.2662) apparently stuck to the column material $[60,83]$, but even if eluted it would have been too large to be efficiently fragmented within the timeframe of a chromatographic peak.

The online version of the original article can be found at http://dx.doi.org/10.1007/s00216-003-2391-2

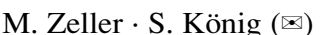

Integrated Functional Genomics,

Interdisciplinary Center for Clinical Research Medical Faculty,

University of Münster,

Von-Esmarch-Str. 56, 48149 Münster, Germany

Tel.: +49-251-8357164, Fax: +49-251-8355651,

e-mail: koenigs@uni-muenster.de 